\title{
REVIEW
}

\section{The action potential: From voltage-gated conductances to molecular structures}

\author{
FRANCISCO BEZANILLA
}

Institute for Molecular Pediatric Sciences, The University of Chicago, CIS Building Room W244. 929 E. $57^{\text {th }}$ Street, Chicago, Illinois, 60637 USA

\section{INTRODUCTION}

The nerve impulse, or action potential, is a fast transient change in the membrane potential of excitable cells that can propagate along axons or muscle fibers and constitutes the bit of information that allows communication in the nervous system. During the action potential, the membrane voltage goes from its resting value of about $-70 \mathrm{mV}$ (negative inside) to about $+30 \mathrm{mV}$ in less than $1 \mathrm{~ms}$ and returns to its resting value in a few milliseconds. Much of what we have learned on the mechanisms of the generation and propagation of the action potential has been using the giant axon of the squid, which allowed the introduction of electrodes into its axoplasm. Thus, Cole and Curtis (1939) were able to measure impedance changes concomitant with the action potential and found that the membrane conductance increased dramatically while the membrane capacitance was essentially constant. Subsequently, Hodgkin and Katz (1949) demonstrated that external $\mathrm{Na}$ ions were required to initiate the action potential, suggesting that the large increase in membrane conductance during the rising phase of the action potential was selective to $\mathrm{Na}$ ions. The detailed studies of the mechanisms in the generation and propagation of the action potential were hampered by the nonlinear nature of the action potential and the propagation itself: the membrane potential is both function of time and distance $(V(x, t))$ and has an extremely nonlinear dependence on the stimulating current. It was the invention of the voltage clamp by Cole (1949) combined with the space clamp of the axon that first allowed the conductance changes to be observed under controlled conditions.

The classical series of papers by Hodgkin, Huxley and Katz and Hodgkin and Huxley culminated in the quantitative description of the axon membrane currents (Hodgkin and Huxley, 1952). In this impressive work, Hodgkin and Huxley demonstrated that the membrane conductance is steeply voltage dependent and were able to separate a conductance that was mainly selective to sodium and another to potassium. The voltage dependence and kinetics of the conductances could explain the generation of the rising and falling phase of the action potential. The empirical description of the sodium and potassium conductances by Hodgkin and Huxley allowed them to set up a system of equations that predicts the action potential time course and all the properties of excitability such as threshold, refractory period, fluxes, etc. The propagation also was predicted by combining these equations with the cable properties of the axon. The work of Hodgkin and Huxley not only forms the basis of the classical electrophysiology that has been in use for more than 50 years, but also gave us insights on many properties of the conductances that have been verified in the same period of time. 
When Eduardo Rojas was starting his independent career in the Laboratorio de Fisiologia Celular in the 1960s, the voltageclamp technique was well established, and he had incorporated the internal perfusion of the axon in his experimental setup, allowing him to control both the internal and external media of the axonal membrane. At that time, it was not clear whether the $\mathrm{Na}$ and $\mathrm{K}$ conductances were in the lipid bilayer or in specialized membrane proteins. He was one of the first to test the effects of proteolytic enzymes on the conductances of the axon (Rojas and Luxoro, 1963). His discovery of the dramatic effect of pronase on the ionic currents under voltage clamp (Rojas and Atwater, 1967a) was one of the first clear indications that proteins were part of the conductance pathways. By blocking the potassium conductance, it was possible to show that the effect of pronase was to block $\mathrm{Na}$ inactivation exclusively (Rojas and Armstrong, 1971; Armstrong et al., 1973), and this result was instrumental in proposing the ball and chain hypothesis of inactivation (Armstrong and Bezanilla, 1977).

\section{WORKING WITH GUAYO}

Eduardo envisioned that the large size of the Chilean squid was a perfect preparation to study ionic currents under voltage clamp while measuring isotopic fluxes simultaneously, thus allowing the measurement of the transport number of the ions crossing the axonal membrane. In 1967, I was extremely excited and honored when Guayo admitted me in his lab to work on my doctoral thesis. He first suggested measuring chloride fluxes during voltage clamp $\left({ }^{36} \mathrm{Cl}\right.$ was an isotope we could get, unlike ${ }^{42} \mathrm{~K}$ ), but we found that their contribution to the resting conductance was quite small, indicating that in the squid axon the leakage current was mainly cationic. During the time I spent in his lab, I learned from Guayo not only how to think, plan, and execute experiments but also many details of voltage clamping, isotope measurements, and above all, the importance of data analysis. Guayo even let me build my own electronics, although in some cases, he did let me know that equipment building could detract from the main purpose of my stay in his lab, which was research.

The simultaneous measurement of ionic currents and fluxes allowed us to test directly whether $\mathrm{Na}$ currents were carried exclusively by $\mathrm{Na}$ after blocking the $\mathrm{K}$ currents. These experiments, done in collaboration with Illani Atwater, confirmed that the transport number of $\mathrm{Na}$ was, indeed, very close to one (Atwater et al., 1969) and also demonstrated that by interrupting the $\mathrm{Na}$ current before it inactivated, the large current tail also was transported exclusively by $\mathrm{Na}$ (Bezanilla et al., 1970a). The next step was to test the $\mathrm{Na}$ hypothesis by measuring the time course of $\mathrm{Na}$ flux directly during the action potential. This experiment required a sampling of the $\mathrm{Na}$ fluxes at different times during the action potential, which is not possible directly because the action potential only lasts a few milliseconds and the time resolution of the flux measurements is in the seconds time scale. The strategy was then to "interrupt" the action potential at different times by switching quickly to voltage clamp so we could measure fluxes at different times as the action potential developed.

The action potential interruption became a project in itself because we realized that new information could be obtained even without measuring fluxes. When the electronic switch (whose design was suggested by Clay Armstrong) is commanded to change from current-clamp to voltage-clamp mode, the measured current can be used to compute the conductance across the membrane at the moment of interruption. Therefore, by interrupting the time course of the action potential at different times, it is possible to obtain the time course of the conductance during the action potential. Furthermore, by sequentially interrupting to the $\mathrm{Na}$ reversal potential, the current measured will be mainly $\mathrm{K}$ current, and then the time course of the $\mathrm{K}$ conductance can be computed during the action potential. On the other hand, by sequentially interrupting to the $\mathrm{K}$ reversal potential, the time course of $\mathrm{Na}$ conductance during the action potential can be obtained (see Fig. 1). These experiments - 
done in collaboration with Bob Taylor, who provided deep insight in the planning and analysis - gave for the first time an experimental demonstration that the $\mathrm{Na}$ and $\mathrm{K}$ conductances follow closely the time course that was predicted by the Hodgkin and Huxley equations (Rojas et al., 1970; Bezanilla et al., 1970b). The main difference of the experimental result using interruption with respect to the Hodgkin and Huxley equations was that there was less overlap of the $\mathrm{Na}$ and $\mathrm{K}$ conductance. The reason for the decreased overlap is the short delay in $\mathrm{K}$ conductance activation in the $\mathrm{n}^{4}$ formulation, a problem that Hodgkin and Huxley were aware of and had mentioned explicitly in their last paper of the series (Hodgkin and Huxley, 1952). Finally, to measure the time course of the $\mathrm{Na}$ flux during the action potential, we combined the action potential interruption technique with simultaneous isotopic flux experiments. The results (Atwater et al., 1970) provided a direct experimental demonstration of the sodium hypothesis of Hodgkin and Huxley.

The action potential interruption technique recently was used to study the $\mathrm{Na}$ and $\mathrm{K}$ conductances of axons from two different squid species, Loligo pealei and Loligo plei. (see Fig. 1). The Woods Hole squid, $L$. pealei, lives in colder waters than $L$. plei, which lives in the Caribbean.
Action potentials are generated and propagated in $L$. plei at temperatures as high as $40^{\circ} \mathrm{C}$ while at temperatures exceeding $28^{\circ} \mathrm{C}$, the action potential in $L$. peale $i$ fails. The main reason for the action potential failure is the acceleration of both conductances by the high temperature that does not allow enough time to the net inward current that depolarizes the cell before the outward $\mathrm{K}$ current starts repolarization. The action potential interruption experiments revealed that, although the $\mathrm{Na}$ conductances are the same in both species, in $L$. plei the $\mathrm{K}$ conductance is significantly reduced with respect to the conductance in L. pealei, thus giving enough time to the $\mathrm{Na}$ conductance to depolarize the membrane before the inward current gets cut short by the outward K current (Rosenthal and Bezanilla, 2002).

\section{SEPARATE PATHWAYS AND SINGLE CHANNELS}

The work of Hodgkin and Huxley (1952) had many predictions and interpretations that had not yet been verified in the 1960s and 1970s. One interpretation of their results was that the $\mathrm{Na}$ and $\mathrm{K}$ conductances were separate pathways. The discovery of tetrodotoxin, TTX (Narahashi et al., 1964) showed that the $\mathrm{Na}$ conductance could be

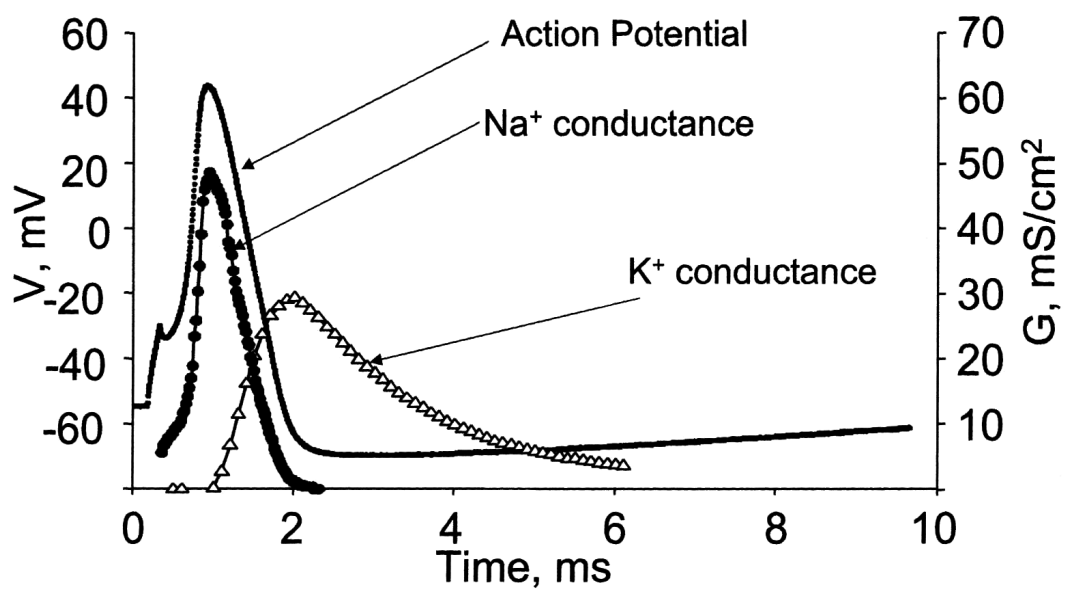

Figure 1. Using the action potential interruption technique, it is possible to obtain the time course of the $\mathrm{Na}$ conductance (by interrupting to the $\mathrm{K}$ reversal potential) and the time course of the $\mathrm{K}$ conductance (by interrupting to the $\mathrm{Na}$ reversal potential). Experiment on Loligo pealei in collaboration with Josh Rosenthal. 
blocked, while the $\mathrm{K}$ conductance remained unaffected. Eduardo made this more evident by demonstrating that TTX not only blocked the inward sodium current but also the outward sodium current in the squid axon (Rojas and Atwater, 1967b). Tetraethylammonium ion (TEA) (Tasaki and Hagiwara, 1957) was found to block the $\mathrm{K}$ conductance without affecting the $\mathrm{Na}$ current. Although these results are quite compelling in support of two separate pathways, there was still the possible explanation of a single pathway that first allows $\mathrm{Na}$ to go through but when the $\mathrm{Na}$ conductance inactivates, the $\mathrm{Na}$ pathway would convert into a $K$ pathway. The results with pronase that showed that the $\mathrm{K}$ conductance was unaffected during inactivation removal ruled out this possibility (Armstrong et al., 1973). Years later, a biochemical demonstration was the purification of the $\mathrm{Na}$ channel protein from eel electroplax (Agnew et al., 1978) and its eventual reconstitution that allowed the recording of $\mathrm{Na}$ currents.

Hodgkin and Huxley (1952) predicted that the conduction was done by discrete units that we now call 'single channels'. The introduction of the patch-clamp technique (Neher and Sakmann, 1976; Hamill et al., 1981) confirmed that the macroscopic sodium ionic currents were produced by discrete single sodium channels (Sigworth and Neher, 1980), and this finding was directly verified for $\mathrm{K}$ channels (Conti and Neher, 1980) and $\mathrm{Na}$ channels (Bezanilla, 1987) in the squid giant axon.

\section{GATING CURRENTS}

Hodgkin and Huxley (1952) also predicted that voltage dependence required the translocation of charge across the membrane that would act as the voltage sensor of the conductance. The movement of that charge should produce a transient current. This putative current is expected to be much smaller than the ionic current through the conductance. In 1971, we undertook a first attempt at measuring these currents in Woods Hole with Clay Armstrong and Eduardo Rojas, but we were unable to see them convincingly in capacity transients recorded on film from axon bathed and perfused with impermeant ions. In 1972, we attempted the experiment again with Clay but Eduardo was not in Woods Hole at that time. To enhance the signal, we had to eliminate the large linear component of the capacity current. For this purpose, we subtracted the currents produced by pulses of opposite polarities, and we used an analog signal averager. In this fashion, we recorded the gating currents of the $\mathrm{Na}$ channel as a transient current that preceded the opening of the $\mathrm{Na}$ conductance (Armstrong and Bezanilla, 1973). Across the Atlantic, Eduardo and Richard Keynes also detected the $\mathrm{Na}$ gating currents in the giant axon of the Plymouth squid (Keynes and Rojas, 1974).

Many of the properties of the experimentally recorded $\mathrm{Na}$ gating currents were in agreement with the predictions from the Hodgkin and Huxley (HH) formulation except for two important differences. In the $\mathrm{HH}$ formulation, there are three independent voltage-sensing particles that move to the active position upon depolarization, generating the gating current, but all three are needed to open the conductance. Thus, upon repolarization it is just enough that one of the particles moves back to close the conductance, which means that the kinetics of the conductance should be three times faster than the kinetics of the gating current. This was not the case: the time constant of gating current upon repolarization was never slower than 1.7 times the time constant of the conductance decrease (Armstrong and Bezanilla, 1974).

The other difference between the $\mathrm{HH}$ formulation and the experimental gating current pertains to inactivation. In the $\mathrm{HH}$ model, the inactivation process is independent from activation and has a separate voltage sensor. The experimentally recorded $\mathrm{Na}$ gating currents did not exhibit the kinetic component expected from the inactivation voltage sensor. Instead, a long depolarizing pulse that produces $\mathrm{Na}$ inactivation decreased the gating current, and the voltage dependence and kinetics of this decrease correlated with the voltage dependence and kinetics of the inactivation of the 
conductance. This charge decrease, termed charge immobilization, was in fact a change in the kinetics of the movement of the gating charge such that it became too slow to be detected. At large negative potentials, the immobilized charge could be seen moving with the kinetics of recovery from inactivation, thus confirming that the charge was immobilized by the inactivation process. These results were in agreement with previous results (Goldman and Schauf, 1972) that indicated a coupling between activation and inactivation. The detailed description of ionic currents and gating currents confirmed that activation and inactivation were coupled such that most of the voltage dependence of inactivation was the result of coupling to the voltage dependence of activation. When inactivation was removed by pronase perfused intracellularly (see above), gating currents were no longer immobilized by depolarization. This result suggested the "ball and chain" mechanism of inactivation, a molecular interpretation of inactivation whereby a tethered region of the channel protein blocks the ion conduction pathway and interacts with the gating charge to impede its movement (Bezanilla and Armstrong, 1977; Armstrong and Bezanilla, 1977).

When the first sequence of a voltagedependent $\mathrm{Na}$ channel (Noda et al., 1984) and, subsequently, a voltage-dependent $\mathrm{K}$ channel (Tempel et al., 1987) were reported, it was a breakthrough that not only confirmed that $\mathrm{Na}$ and $\mathrm{K}$ channels were separate entities but also opened the opportunity of modifying the structure by introducing mutations and studying them in isolation, using expression systems such as the Xenopus oocyte membrane. Thus, Zagotta et al. (1990) showed that the Nterminal part of Shaker was responsible for inactivation because its deletion eliminated inactivation and the reintroduction of the peptide restored inactivation. The high density of channels expressed on the oocyte made it possible to record gating currents from Shaker K channels with very high resolution and little contamination of other non-linear currents. It was then possible to show that the "ball and chain" indeed produced charge immobilization that could be eliminated by using the channel without the N-terminal region (Bezanilla et al., 1991). Detailed studies of K-gating currents were now possible and generated kinetic models that reproduced ionic and gating currents and gave insight on the physical mechanisms that generated voltage dependence (Bezanilla et al., 1994; Zagotta el al., 1994). Schoppa et al. (1992) measured the total gating charge and the total number of channels and were able to compute the charge per channel that is necessary to open the conductance and found a total of $13 \mathrm{e}_{0}$. It is important to note that these measurements do not give the number of charges but the total charge times the fraction of the field they traverse.

\section{THE GATING CHARGE RESIDUES}

The primary sequences of voltage-dependent channels showed a region of the protein that contained a sequence of basic residues (arginines or lysines) separated by two hydrophobic residues. This sequence was in the fourth putative transmembrane segment (S4), and there were four S4 subunits or domains per channel. It was immediately postulated that the voltage sensor resided in the S4 segment because it insinuated a simple mechanism of charge translocation across the membrane that would give origin to the gating currents. Several attempts were made to prove this point by neutralizing the charges (mutating the arginine or lysine to a non-charged amino acid) and observing the effects on the ionic currents. It was clear that neutralization of the S4 charges had high impact in the voltage dependence of the conductance but so did other mutations that did not change charged amino acids. The unequivocal way to prove that a charged amino acid contributes to the gating current is to show that upon its neutralization (point mutation) the total charge per channel is decreased. This was done by Aggarwal and MacKinnon (1996) and by Seoh et al. (1996) when they demonstrated that the first four most extracellular arginines of the S4 segment in Shaker $\mathrm{K}^{+}$channel were directly involved in the gating charge movement. In addition, a negative residue (E293) in the S2 segment also was shown to participate in the 
gating charge (Seoh et al., 1996). Interestingly, the neutralization of several charged residues in Shaker decreased the gating charge by more than four elementary charges, indicating that the presence of a gating charged residue modifies the local electric field seen by the other charges.

Knowing which part of the channel molecule generates the gating current was a significant advance that began transforming our models of gating from black boxes to models based on a physical reality. However, a complete physical model of voltage activation requires a description of the actual movements that the gating charges undergo and how they interact with the electric field. This information, still incomplete, has been gathered with a multitude of biophysical and structural techniques.

CHANGES OF EXPOSURE OF THE GATING CHARGES UPON VOLTAGE CHANGES

One way to probe for movements of the charges when the field is changed is by testing their possible changes of exposure to extra and intracellular medium. The pioneer work of Horn and collaborators (Yang and Horn, 1995) showed for the first time that indeed such exposure change occurs. By mutating the sought residues to cysteine and probing them with cysteinereactive reagents from either side of the membrane, they found that the reactivity depended on the voltage applied.

Another way to probe for movement is to test for changes in accessibility of protons to the charged residues. As the arginine has a very high $\mathrm{pK}_{\mathrm{a}}$, this test can be done by replacing the arginines by histidine one at a time. If there is a change in exposure to the intra- or extracellular solution concomitant with the conformational change induced by voltage, then in addition to the gating current changes, one also may observe a proton current under the appropriate $\mathrm{pH}$ gradient. In fact, it was found that the first four most extracellular charges in S4 indeed were exposed alternatively to the inside at hyperpolarized potentials and to the outside at depolarized potentials. The second, third and fourth charged residues were found to become proton transporters when substituted by histidine (Starace et al., 1997; Starace and Bezanilla, 2001). The first charge became a proton pore at hyperpolarized potentials (Starace and Bezanilla, 2004), and the fourth charge became a proton pore at extreme depolarized potentials, while the fifth and sixth charges were not titrateable by protons (Starace and Bezanilla, 2001), as expected due to the fact that they do not participate in gating charge movement (Seoh et al., 1996). The presence of the proton pore delineated a voltage sensor that had a very concentrated field in which the charges did not need to move a large distance. The concentrated field was experimentally inferred by changing the ionic strength (Islas and Sigworth, 2001) and measured directly using an electrochromic probe attached to specific points of the channel molecule (Asamoah et al., 2003).

\section{CONFORMATIONAL CHANGES DETECTED BY}

FLUORESCENCE

By labeling specific sites of the channel protein with fluorescent probes, it is possible to measure local conformational changes by observing the changes in fluorescence as the channel is cycled between the closed and open state (Mannuzzu et al., 1996; Cha and Bezanilla, 1997). Most of the changes in fluorescence have been traced to changes in quenching of the fluorophore as the molecule undergoes conformational changes (Cha and Bezanilla, 1997, 1998). One of the most interesting results obtained with this technique was the identification of the unique function of each of the domains in the Na channel. Thus, it was demonstrated that charge immobilization induced during inactivation was associated with changes in domains III and IV but had no influence in domains I and II (Cha et al., 1999b). This explained at the molecular level the finding that inactivation immobilized only $2 / 3$ of the gating charge (Armstrong and Bezanilla, 1977). The S4 of domain IV also was found to have a slower kinetics than the movement of S4 segments in domains I, II and III and also exhibited a lag, suggesting that it moves 
after the other three domains. In addition, the time course of the fluorescence changes in DI, DII and DIII correlated with the fast component of the $\mathrm{Na}$ gating current, while the kinetics of fluorescence in DIV correlated with the slow component of the gating current (Chanda and Bezanilla, 2002). This also solved the old unanswered question of the origin of the slow component in the gating current that was postulated to be responsible for a second open state (Armstrong and Bezanilla, 1977). The results of fluorescence kinetics are consistent with a specialized function of DIV-S4 that prepares the site for the docking of the inactivation gate and opens the possibility that this segment is not directly involved in opening the conduction pore, as suggested by Sheets and Hank (1995). As each one of the domains can be studied separately in the $\mathrm{Na}$ channel, the effect of a perturbation in one domain can be studied in other domains using fluorescence (Chanda et al., 2004). This approach has revealed that the $\mathrm{S} 4$ segments show positive cooperativity between domains, and this result helps in explaining why the $\mathrm{Na}$ channel is much faster than the $\mathrm{K}$ channel, a requirement to elicit an action potential.

Fluorescence resonance energy transfer (FRET) is a powerful technique that allows the estimation of distances between a donor fluorophore and acceptor molecule. Thus, by labeling the channel molecule with a donor and an acceptor, it is possible to measure the position and change in position of the labels as the channel cycles from closed to open. A variant of this technique uses terbium, which is a lanthanide that emits isotropically and with a time constant in the millisecond range (as opposed to nanonsecond for organic fluorophores). These are important advantages that give high accuracy in distance estimates, better than 10\% (Selvin, 2002). Two LRET studies of distance changes in the Shaker K channel have revealed that during depolarization the S4 undergoes very little radial movement nor translation across the membrane. In the first study (Cha et al., 1999b), the distance between subunits was measured in the closed and open state revealing that radial changes did not exceed $3 \AA$ A. A similar study done with conventional FRET also revealed minimum radial changes (Glauner et al., 1999). In the second LRET study (Posson et al., 2005) the donor was located in different positions in the channel (including S3, S3-S4 linker and S4), and the acceptor was located in a toxin that has a high affinity for the pore of Shaker. This study revealed that changes in distance did not exceed $2 \AA$, indicating a minimum translation of the S4 segment across the membrane.

The small translation of S4 is not consistent with the original helical screw model of activation (Catterall, 1986; Durell and Guy, 1992) nor with the most recent paddle model that translates most of the S4 segment from the intracellular to the extracellular solution (Jiang et al., 2003b). However, the small translation of S4 was consistent with the notion that the field was concentrated and that the charged residues were in water-filled crevices penetrating the protein core (Bezanilla, 2000).

One more FRET experiment confirmed the small translation of the S4 segment. The acceptor used was a non-fluorescent hydrophobic ion that sits in the lipid bilayer and translocates from one leaflet to the other depending on the membrane potential, very much like gating charges embedded in the bilayer. The donor was a fluorophore located in different positions of the channel (the pore and S4). The time course of the fluorescence of the donor depends on the position of the acceptor such that if both donor and acceptor translocate, the fluorescence show a transient behavior, while if the donor (in S4) does not cross the membrane, the fluorescence change is monotonic. All residues tested showed monotonic changes, confirming that the S4 segment undergoes minimal movement across the membrane (Chanda et al., 2005).

\section{A MODEL OF THE VOLTAGE SENSOR}

The results of histidine scanning, electrochromic probes, fluorescence, FRET, and LRET give enough constraints to propose a general model of activation of a voltage-gated channel. The structural details of the molecule were obtained from the 
partial crystal structure of the S1-S4 of KvAP (Jiang et al., 2003a). KvAP is a voltage-gated channel from an archebacteria that was crystallized by Jiang et al. (2003a) but showed a clearly non-native conformation. However, the same authors obtained a crystal of the voltage sensor of KvAP that was a useful starting point to build a model of the channel with all the above-mentioned results and several other biophysical constraints. One important additional constraint for the open state was a high affinity metal bridge between an engineered histidine replacing the first charge of S4 and another engineered histidine in the pore region of Shaker (Laine et al., 2003). An important constraint of the closed state was the proton pore formed when the first charged residue is replaced by histidine. A schematic representation of the proposed model (Chanda et al., 2005) is shown in Figure 2. The salient features of this model are that most of the charged groups of the arginines reside in a large and deep internally connected water crevice in the closed state and a shallower externally connected water crevice in the open state. Thus in the closed state the outermost arginine is in the concentrated electric field that, when changed, moves exerting a torque on the entire S4 segment that undergoes a change in tilt that eliminates the internal water crevice, thus moving the charges to an externally created water crevice.

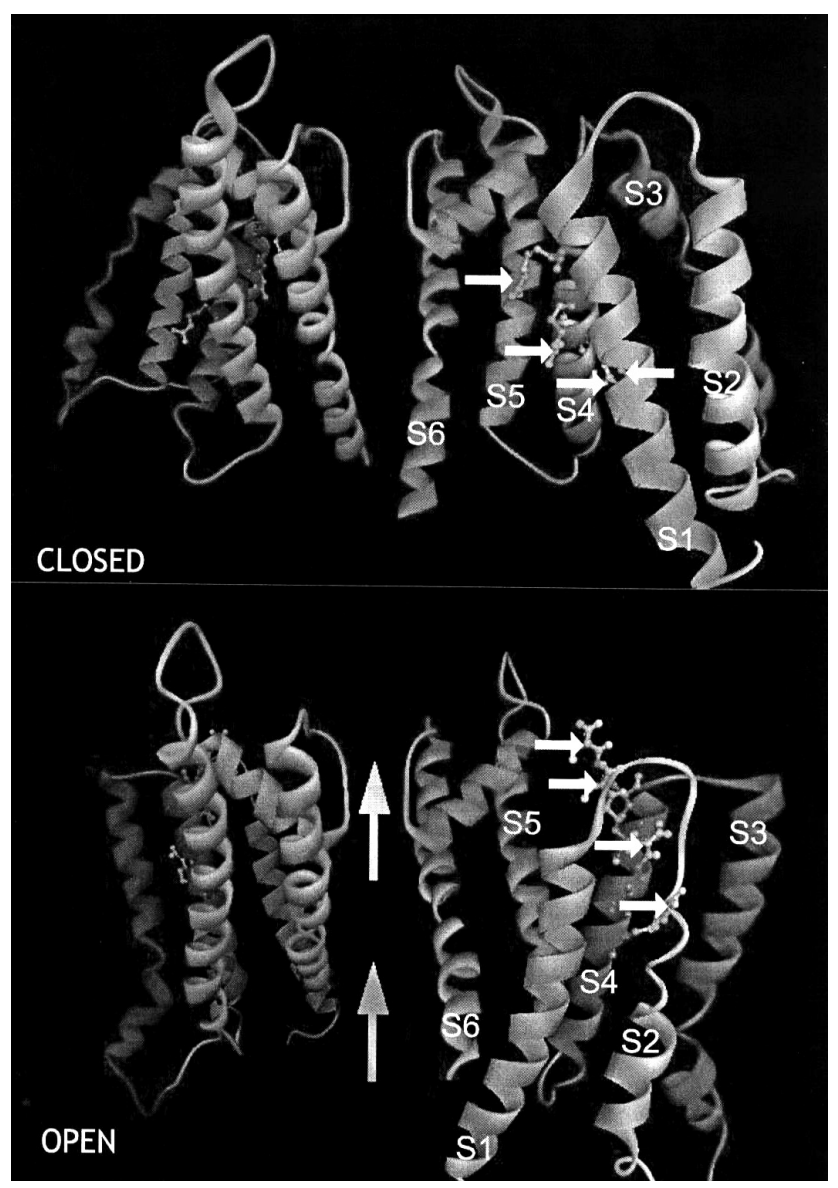

Figure 2. Molecular models of the closed and open states of Shaker K channel presented by Chanda et al. (2005). For clarity, only two of the four subunits are shown. Horizontal arrow point to the charged group of the arginines. Vertical arrows picture the flow of $\mathrm{K}$ through the open pore. (A detailed simulation of the ionic and gating currents with this model can be found at http: // nerve.bsd.uchicago.edu/model/rotmodel.html). 
The change in conformation as proposed by the two structures can be used to compute the net charge movement using the PoissonBoltzmann equations (Roux, 1997), giving a total of $13 \mathrm{e}_{0}$ (Chanda et al., 2005), in excellent agreement with the experimentally measured value (Schoppa et al., 1992). How does the channel open? The model suggests that the change in position of the S4 segment couples to the intracellular end of S5 that as it moves, allows the expansion of the intracellular side of S6 that can spread apart opening the pore. This particular conformation recently has been seen in the new crystal structure of Kv1.2 (Long et al., 2005). It is interesting to note that the proposed structure of the open state in Chanda et al. (2005), which was obtained with biophysical data from Shaker and partial crystal structures from KvAP, is very similar to the Kv1.2 crystal structure, which corresponds to an open inactivated state.

\section{CONCLUSION}

The generation and propagation of the action potential depends on the interplay of the sodium and potassium voltage dependence conductances. The original Hodgkin and Huxley formulation made specific predictions as to how the voltage can change the conductance. The progress in membrane biochemistry, molecular biology, structural techniques, and spectroscopy have confirmed many of the basic assumptions of Hodgkin and Huxley and have made possible the evolution from a black box description to a molecular description of excitability. The ultimate goal is a quantitative physical description of all the states and kinetic transitions between states of the channel molecule. Spectroscopy combined with crystallography and molecular computational techniques are the tools that are expected to help in realizing this goal.

\section{REFERENCES}

AGGARWAL SK, MACKINNON R (1996) Contribution of the $\mathrm{S} 4$ segment to gating charge in the Shaker $\mathrm{K}^{+}$ channel. Neuron 16: 1169-1177

AGNEW WS, LEVINSON SR, BRABSON JS, RAFTERY
MA (1978) Purification of the tetrodotoxin-binding component associated with the voltage-sensitive sodium Channel from Electrophorus electricus electroplax membranes. Proc Nat Acad Sci USA 75: 2602-2610

ARMSTRONG CM, BEZANILLA F (1973) Currents related to movement of the gating particles of the sodium channels. Nature 242: 459-461

ARMSTRONG CM, BEZANILLA F (1974) Charge movement associated with the opening and closing of the activation gates of the sodium channels. J Gen Physiol 63: 533-552

ARMSTRONG CM, BEZANILLA F (1977) Inactivation of the sodium channel. II. Gating current experiments. J Gen Physiol 70: 567-590

ARMSTRONG CM, BEZANILLA F, ROJAS E (1973) Destruction of sodium conductance inactivation in squid axons perused with pronase. J Gen Physiol 62: 375-391

ASAMOAH OK, WUSKELL JP, LOEW LM, BEZANILLA F (2003) A fluorometric approach to local electric field measurements in a voltage-gated ion channel. Neuron 37: 85-97

ATWATER I, BEZANILLA F, ROJAS E (1969). Sodium influxes in internally perfused squid giant axon during voltage clamp. J Physiol (Lond) 201: 657-664

ATWATER I, BEZANILLA F, ROJAS E (1970) Time course of the sodium permeability change during a single membrane action potential. J Physiol (Lond) 211: 753-765

BEZANILLA F (1987) Single sodium channels from the squid giant axon. Biophys J 52: 1087-1090

BEZANILLA F (2000) The voltage sensor in voltagedependent channels. Physiological Reviews 80: 555592

BEZANILLA F, ARMSTRONG CM (1977) Inactivation of the sodium channel. I. Sodium current experiments. J Gen Physiol 70: 549-566

BEZANILLA F, PEROZO E, PAPAZIAN DM, STEFANI E (1991) Molecular basis of gating charge immobilization in Shaker potassium channels. Science 254: 679-683

BEZANILLA F, PEROZO E, STEFANI E (1994) Gating of Shaker $\mathrm{K}^{+}$channels. II. The components of gating currents and a model of channel activation. Biophys $\mathbf{J}$ 66: 1011-1021

BEZANILLA F, ROJAS E, TAYLOR RE (1970a) Time course of the sodium influx in squid giant axon during a single voltage clamp pulse. J Physiol (Lond) 207: 151-164

BEZANILLA F, ROJAS E, TAYLOR RE (1970b) Sodium and potassium conductance changes during a membrane action potential. J Physiol (Lond) 211: 729751

CATERALL WA (1986) Molecular properties of voltagesensitive sodium channels. Annu Rev Biochem 55: 953-985

CHA A, BEZANILLA F (1997) Characterizing voltagedependent conformational changes in the Shaker $\mathrm{K}^{+}$ channel with fluorescence. Neuron 19: 1127-1140

CHA A, BEZANILLA F (1998) Structural implications of fluorescence quenching in the Shaker $\mathrm{K}^{+}$channel. J Gen Physiol 112: 391-408

CHA A, SNYDER G, SELVIN PR, BEZANILLA F (1999a) Atomic scale movement of the voltage-sensing region in a potassium channel measured via spectroscopy. Nature 402: 809-813

CHA A, RUBEN PC, GEORGE AL, FUJIMOTO E, BEZANILLA F (1999b) Voltage sensors in domains III and IV, but not I and II, are immobilized by $\mathrm{Na}^{+}$ channel fast inactivation. Neuron 22: 73-87 
CHANDA B, BEZANILLA F (2002) Tracking voltagedependent conformational changes in skeletal muscle sodium channel during activation. J Gen Physiol 120: 629-645

CHANDA B, ASAMOAH OK, BEZANILLA F (2004) Coupling interactions between voltage sensors of the sodium channel as revealed by site-specific measurements. J Gen Physiol 123: 217-230

CHANDA B, OK ASAMOAH, R BLUNCK, B ROUX and F BEZANILLA. (2005) Gating charge displacement in voltage-gated channels involves limited transmembrane movement. Nature 436: 852-856.

COLE KS (1949) Dynamic electrical characteristics of the squid axon membrane. Arch Sci Physiol 3: 253-258

COLE KS, CURTIS HJ (1939) Electric impedance of the squid giant axon during activity. J Gen Physiol 22: 649-670

CONTI F, NEHER E (1980) Single channel recording of $\mathrm{K}+$ currents in squid axons. Nature 285: 140-143

DURELL SR, GUY HR (1992) Atomic scale structure and functional models of voltage-gated potassium channels. Biophys J 62: 238-250

GLAUNER KS, MANNUZZU LM, GANDHI CS, ISACOFF EY (1999) Spectroscopic mapping of voltage sensor movements in the Shaker potassium channel. Nature 402: 813-817

GOLDMAN L, SCHAUF CL (1972) Inactivation of the sodium current in Myxicola giant axons. Evidence of coupling to the activation process. J Gen Physiol 59: 659-675

HAMILL OP, MARTY A, NEHER E, SAKMANN B, SIGWORTH FJ (1981) Improved patch-clamp techniques for high-resolution current recording from cells and cell-free membrane patches. Pflugers Arch 391: $85-100$

HODGKIN AL, KATZ B (1949) The effect of sodium ions on the electrical activity of the giant axon of the squid. J Physiol 108: 37-77

HODGKIN AL, HUXLEY AF (1952) A quantitative description of membrane current and its application to conduction and excitation in nerve. J Physiol 117: 500544

ISLAS LD, SIGWORTH FJ (2001) Electrostatics and the gating pore of Shaker potassium channels. J Gen Physiol 117: 69-89

JIANG Y, LEE A, CHEN J, CADENE M, CHAIT BT, MACKINNON R (2002) The open pore conformation of potassium channels. Nature 417: 523-526

JIANG Y, LEE A, CHEN J, RUTA V, CADENE M, CHAIT BT, MACKINNON R (2003) X-ray structure of a voltage-dependent $\mathrm{K}(+)$ channel. Nature 423: 3341

KEYNES RD, ROJAS E (1974) Kinetics and steady-state properties of the charged system controlling sodium conductance in the squid giant axon. J Physiol 239393434.

LAINE M, LIN MC, BANNISTER JP, SILVERMAN WR, MOCK AF, ROUX B, PAPAZIAN DM (2003) Atomic proximity between S4 segment and pore domain in Shaker potassium channels. Neuron 39: 467-481

LONG SB, CAMPBELL EB, MACKINNON R (2005) Crystal structure of a mammalian voltage-dependent Shaker family $\mathrm{K}^{+}$channel. Science 309: 897-903

MANNUZZU LM, MORONNE MM, ISACOFF EY (1996) Direct physical measure of conformational rearrangement underlying potassium channel gating. Science 271: 213-216

NARAHASHI T, MOORE JW, SCOTT WR (1964) Tetrodotoxin blockage of sodium conductance increase in lobster giant axons. J Gen Physiol 47: 965-974
NEHER E, SAKMANN B (1976) Single-channel currents recorded from membranes of denervated frog muscle fibres. Nature 260: 799-802

NODA M, SHIMIZU S, TANABE T, TAKAI T, KAYANO T, IKEDA T, TAKAHASHI H, NAKAYAMA H, KANOAKA Y, MINAMINO N (1984) Primary structure of Electrophorus electricus sodium channel deduced from cDNA sequence. Nature 312: 121-127

POSSON DJ, Ge P, MILLER C, BEZANILLA F, SELVIN PR (2005) Small vertical movement of a $\mathrm{K}^{+}$channel voltage sensor measured with luminescence energy transfer. Nature 436: 848-851

ROJAS E, ARMSTRONG CM (1971) Sodium conductance activation without inactivation in pronase-perfused axons. Nature New Biology 229: 117

ROJAS E, ATWATER I (1967a) Blocking of potassium currents by pronase in perfused giant axons. Nature 215: 850

ROJAS E, ATWATER I (1967b) Effect of tetrodotoxin on the early outward currents in perfused giant axons. PNAS 57: 1350-1355

ROJAS E, BEZANILLA F, TAYLOR RE (1970) Demonstration of sodium and potassium conductance changes during a nerve action potential. Nature 225: 747-748

ROJAS E, LUXORO M (1963) Micro-injection of trypsin into axons of squid. Nature 199: 78-79

ROSENTHAL JJ, BEZANILLA F (2002) A comparison of propagated action potentials from tropical and temperate squid axons: Different durations and conduction velocities correlate with ionic conductance levels. J Exp Biol 205: 1819-30

ROUX B (1997) Influence of the membrane potential on the free energy of an intrinsic protein. Biophys $\mathrm{J} 73$ : 2980-2989

SCHOPPA NE, MCCORMACK K, TANOUYE MA, SIGWORTH FJ (1992) The size of gating charge in wild-type and mutant shaker potassium channels. Science 255: 1712-1715

SELVIN PR (2002) Principles and biophysical applications of luminescent lanthanide probes. Ann Rev Biophysics and Biomolec Struct 31: 275-302

SEOH SA, SIGG D, PAPAZIAN DM, BEZANILLA $F$ (1996) Voltage-sensing residues in the $\mathrm{S} 2$ and $\mathrm{S} 4$ segments of the Shaker $\mathrm{K}^{+}$channel. Neuron 16: 11591167

SHEETS MF, HANCK DA (1995) Voltage-dependent open-state inactivation of cardiac sodium channels: Gating current studies with Anthopleurin-A toxin. J Gen Physiol 106: 617-640

SIGWORTH FJ, NEHER E (1980) Single $\mathrm{Na}^{+}$channel currents observed in cultured rat muscle cells. Nature 287: 447-449

STARACE DM, STEFANI E, BEZANILLA F (1997) Voltage-dependent proton transport by the voltage sensor of the Shaker $\mathrm{K}^{+}$channel. Neuron 19: 13191327

STARACE DM, BEZANILLA F (2001) Histidine scanning mutagenesis of basic residues of the S4 segment of the Shaker $\mathrm{K}^{+}$channel. J Gen Physiol 117: 469-490

STARACE DM, BEZANILLA F (2004) A proton pore in a potassium channel voltage sensor reveals a focused electric field. Nature 427: 548-552

TASAKI I, HAGIWARA S (1957) Demonstration of two stable potential states in the squid giant axon under tetraethylammonium chloride. J Gen Physiol 40: 859885

TEMPEL BL, PAPAZIAN DM, SCHWARTZ TL, JAN YN, JAN LY (1987) Sequence of a probable potassium 
channel component encoded at Shaker locus in Drosophila. Science 237: 770-775

YANG N, HORN R (1995) Evidence for voltage-dependent S4 movement in sodium channels. Neuron 15: 213-218

ZAGOTTA WN, HOSSI T, ALDRICH R (1990) Restoration of inactivation in mutants of Shaker potassium channels by a peptide derived from ShB. Science 250: 568-571

ZAGOTTA WN, HOSHI T, DITTMAN J, ALDRICH R

(1994) Shaker potassium channel gating III: Evaluation of kinetic models for activation. J Gen Physiol 103: 321-362 
